\title{
EFECTOS DE ACTIVIDADES FONOLÓGICAS EN EL VOCABULARIO, LAS HABILIDADES PSICOLINGÜÍSTICAS Y LOS PROCESOS LECTORES DE NIÑOS DE PRIMER GRADO
}

\author{
Iris Xóchitl Galicia Moyeda*, Francisco Javier Robles Ojeda, Alejandra Sánchez Velasco \\ Facultad de Estudios Profesionales Iztacala, \\ Universidad Nacional Autónoma de México
}

Recibido, julio 1/2014

Concepto de evaluación, diciembre 2/2014

Aceptado, abril 21/2015
Referencia: Galicia Moyeda, I.X., Robles Ojeda, F.J. \& Sánchez Velasco, A. (2015). Efectos de actividades fonológicas en el vocabulario, las habilidades psicolingüísticas y los procesos lectores de niños de primer grado. Acta Colombiana de Psicología, 18 (2), 29-40. DOI: 10.14718/ACP.2015.18.2.3

Resumen

\begin{abstract}
La adquisición de la lectoescritura involucra diversos procesos que pueden estimularse en contextos cotidianos, como el hogar, y que también pueden ser favorecidos por la instrucción escolar. Se trabajó con un grupo de niños de primer grado de educación primaria en el contexto escolar durante seis meses, realizando actividades que favorecieran el procesamiento fonológico con el objetivo de observar su impacto en la conciencia fonológica, sus habilidades psicolingüísticas, su vocabulario y sus habilidades lectoras. Los resultados de la $t$ de Student revelan, de manera general, que tales actividades beneficiaron la conciencia fonológica, el vocabulario y algunas de las habilidades psicolingüísticas. Posteriormente, se identificaron los niños que al final del ciclo escolar habían adquirido la lectoescritura y los que no. Los resultados de la U de Mann-Whitney y de la rho de Spearman sugieren que, a diferencia del vocabulario y las habilidades psicolingüísticas, el nivel de la conciencia fonológica con el cual los niños inician la instrucción lectora, pudo ser un elemento importante para tal logro. También se revela que los niños no lectores tienen un desempeño más bajo en la ejecución de tareas que involucran el canal auditivo, que en las del canal visual. Palabras clave: procesamiento fonológico, lectoescritura, habilidades psicolingüísticas, vocabulario.
\end{abstract}

\section{EFFECTS OF PHONOLOGICAL ACTIVITIES ON VOCABULARY, PSYCHOLINGUISTIC ABILITIES AND READING PROCESSES IN FIRST GRADE CHILDREN}

\begin{abstract}
Literacy acquisition involves different processes that can be stimulated in everyday contexts like home and that can also be favored by schooling. Work was carried out with a group of first grade children at a school setting during six months, through activities favoring phonological processing, with the aim of observing their impact on the phonological awareness, psycholinguistic abilities, and vocabulary and reading skills in these children. Generally, results of the Student's-t test reveal that such activities encourage phonological awareness, vocabulary and some psycholinguistic abilities. A distinction was subsequently drawn between children who had acquired literacy towards the end of the school year and those who had not done so. Results from Mann-Whitney's U and Spearman's rho suggest that, unlike vocabulary and psycholinguistic abilities, the level of phonological awareness with which children start their reading instruction could have been an important element in such an achievement. It is also revealed that, in performing tasks involving the auditory channel, the performance of children who do not read is lower than in tasks involving the visual channel.

Key words: phonological processing, literacy, psycholinguistic abilities, vocabulary.
\end{abstract}

\footnotetext{
* Av. de los Barrios 1, UIICSE 2do piso. Los Reyes Ixtacala, Tlalnepantla, Edo. de Méx., c.p. 54090. Tel: (52) 55 5561 0758. iris@unam. $\mathrm{mx}$
} 


\title{
EFEITOS DE ATIVIDADES FONOLÓGICAS NO VOCABULÁRIO, NAS HABILIDADES PSICOLINGUÍSTICAS E NOS PROCESSOS LEITORES DE CRIANÇAS DA PRIMEIRA SÉRIE
}

Resumo

\begin{abstract}
A aquisição da lecto-escritura envolve diversos processos que podem estimular-se em contextos cotidianos, como o lar, e que também podem ser favorecidos por a instrução escolar. Trabalhou-se com um grupo de crianças da primeira série da educação primária no contexto escolar durante seis meses, realizando atividades que favorecessem o processamento fonológico com o objetivo de observar seu impacto na consciência fonológica, suas habilidades psicolinguísticas, seu vocabulário e suas habilidades leitoras. Os resultados do $t$ de Student revelam, de maneira geral, que essas atividades beneficiaram a consciência fonológica, o vocabulário e algumas das habilidades psicolinguísticas. Posteriormente, identificaram-se as crianças que no final do ciclo escolar haviam adquirido a lecto-escritura e as que não. Os resultados do U de Mann-Whitney e do rho de Spearman sugerem que, a diferença do vocabulário e das habilidades psicolinguísticas, o nível da consciência fonológica com o qual as crianças começam a instrução leitora, pôde ser um elemento importante para esse sucesso. Revela-se também as crianças não leitores têm um desempenho mais baixo na execução de tarefas que envolvem o canal auditivo, que nas do canal visual.

Palavras chave: processamento fonológico, lecto-escritura, habilidades psicolinguísticas, vocabulário.
\end{abstract}

El proceso de aprendizaje de la lectura comienza mucho antes de recibir una instrucción lectora formal, pues fuera del contexto escolar el niño va teniendo diversas interacciones con el lenguaje oral y escrito. Las diferentes prácticas realizadas cotidianamente en el hogar y en otros contextos, educativos o no, posibilitan que el niño realice discriminaciones cada vez más finas de los elementos constitutivos del lenguaje oral que lo conducirán, entre otras conductas, a segmentar las palabras en sílabas, a reconocer el inicio de la sílaba y la rima al final de la palabra; posteriormente podrá segmentar la palabra en sus fonemas. Estas operaciones que el niño realiza al usar los sonidos del lenguaje hablado le resultan sumamente útiles cuando está aprendiendo a decodificar el lenguaje escrito. El dominio de esas operaciones en las diferentes unidades del lenguaje oral es lo que se conoce como procesamiento fonológico. De acuerdo con Passenger, Stuart y Terrel (2003), las habilidades de procesamiento fonológico se refieren al uso de la información basada en la estructura sonora del lenguaje oral para procesar el habla y el lenguaje escrito. Una de las habilidades incluidas en tal procesamiento es la conciencia fonológica, la cual es definida como la habilidad para reconocer que el habla puede subdividirse en unidades menores que la palabra y que pueden ser manipuladas.

$\mathrm{Si}$ bien en la literatura se reconocen distintas conceptualizaciones de este término, existe el consenso de que se ven implicadas habilidades de discriminación y análisis auditivo gracias a las cuales se llega al reconocimiento y empleo de distintas unidades lingüísticas, en especial de los fonemas, y que dicha habilidad es de suma importancia en el aprendizaje lector (Lorenzo, 2001; Cuadro \& Trías, 2008, Defior, 2008). De acuerdo con la discriminación y manejo de las unidades lingüísticas que realiza el individuo, se reconocen tres tipos de conciencia fonológica (Treiman, 1991): a) conciencia silábica, que se refiere a la habilidad para reconocer, manipular y segmentar las sílabas que componen la palabra; b) conciencia intrasilábica, que es la habilidad de segmentar la sílaba es sus componentes de comienzo y rima, y c) conciencia fonémica, que es la habilidad para identificar los fonemas de las palabras y segmentar estas en dichas unidades y poder manipular los fonemas.

El que los niños reconozcan que las palabras pueden descomponerse en unidades menores, como los fonemas, les facilita el acceso a la lectura (Savage \& Carles, 2004; Cuadro \& Trías, 2008), dado que al distinguir los fonemas que conforman las palabras, la tarea de correspondencia entre grafía y sonido, que se establece al leer y escribir, resulta más sencilla de realizar. Aun cuando algunas habilidades de la conciencia fonológica se desarrollan antes del aprendizaje formal de la lectura, el propio aprendizaje del sistema alfabético contribuye al desarrollo de la conciencia fonológica, en especial en lo que toca a la conciencia fonémica, estableciéndose una relación recíproca (Jiménez \& Ortiz, 2000; Lorenzo, 2001; Bravo, 2004; Defior, 2008).

Se han estimado otros aspectos del lenguaje oral que podrían incidir en el proceso de la adquisición de la lectoescritura. En este trabajo se tiene interés en las habilidades psicolingüísticas y en el vocabulario. Con respecto a las primeras, Kirk, McCartey y Kirk (1961) identificaron una serie de habilidades orales establecidas en los contextos naturales de los niños, y que su escasa promoción puede desembocar en problemas del aprendizaje escolar. Se han diseñado exitosamente entrenamientos fundamentados en tales habilidades (Kavale, 1981), y recientemente hay evidencia de que favorecen el nivel de acceso a la lectoescritura. De tal suerte que se considera que el aprendizaje de 
la lectoescritura se fundamenta en un desarrollo óptimo del lenguaje oral, tanto a nivel comprensivo como expresivo, potenciado por experiencias previas al proceso formal de su enseñanza y favorecido por diversas actividades escolares (Hesketh, Adams \& Nightingale, 2000; Wood \& Terrell, 2000; Poe, Burchinal \& Roberts, 2004). Con relación a esta apreciación podría preguntarse en qué medida se encuentran relacionadas las habilidades psicolingüísticas y las fonológicas.

Otro aspecto atendido es el vocabulario receptivo, ya que se considera que en el reconocimiento adecuado de una palabra escrita, el niño debe recuperar su nombre y su significado. Hay estudios que encuentran una relación estrecha entre el nivel de desarrollo del vocabulario con el nivel de la lectoescritura y la lectura de comprensión (Herrera, 2005; Santiuste \& López, 2005), e incluso se considera como un buen predictor para el fracaso o el éxito escolar (Moraleda, 1991; Dalurzo \& González, 2004; González \& Delgado, 2009; Palomino, 2011). El vocabulario del niño también es estimulado por las prácticas cotidianas no escolares realizadas por las madres y conocidas como técnicas de enseñanza implícitas (Ninio \& Bruner, 1978 y West, Stanovich \& Mitchell, 1993) que promueven también las habilidades lingüísticas necesarias para la alfabetización (Valdez_Menchaca \& Whitehurst, 1992), por lo que Sénéchal, Thomas y Monker (1995) sugieren que esas habilidades posibilitan una representación fonológica de las nuevas palabras, la cual le permite al niño extraer información y atribuirles un significado para identificarlas y emplearlas posteriormente en diversas actividades como la lectoescritura.

Con respecto a la apreciación anterior, la relación entre el nivel de vocabulario y las habilidades fonológicas ha sido poco estudiada. Se ha considerado que existe una estrecha relación entre estos elementos porque es más fácil identificar los componentes de una palabra si esta le resulta familiar al individuo que está aprendiendo a leer (Arnaiz, Castejón \& Ruiz, 2002; Flórez-Romero, Restrepo \& Schwanenflugel, 2009 y Stahl \& Yaden, 2004). Walley, Metsala y Garlock (2003) proponen el modelo de reestructuración léxica, según el cual la representación fonológica que se desarrolla de la palabra influye en el posterior reconocimiento oral de esta. Al aprender palabras que suenan de forma similar, es necesario segmentarlas para diferenciarlas. Es decir, el hecho de reconocer oralmente una palabra que implica tener una imagen sonora de esta, favorece la identificación de los fonemas de dicha palabra. Los citados investigadores encontraron que el rendimiento de los niños en las tareas de procesamiento fonológico se relaciona con la habilidad que tienen para reconocer oralmente la palabra, por lo que sugieren que la conciencia fonémica, el vocabulario recep- tivo y la memoria verbal explican la posterior habilidad en el acceso a la lectura. En esta misma línea se han encontrado asociaciones entre el vocabulario receptivo, tareas específicas de conciencia fonológica (emparejar palabras y aislar sonidos) y pruebas simples de lectura (Márquez \& De la Osa, 2003); entre vocabulario receptivo y la identificación de rimas y de sílabas (Diuk, Borzone \& Ledesma, 2010), y entre vocabulario receptivo y emparejamiento de imágenes según su rima (Macià, 2012). Esto sugiere que el léxico es el punto de partida para empezar a establecer buenas destrezas fonológicas, pero no para profundizar en estas habilidades que requieren de una enseñanza más intencional. No obstante, existen propuestas de que la tarea fonológica al leer no estaría relacionada con el desarrollo del vocabulario en un inicio, sino hasta que ya se domina por completo la alfabetización (Vargas \& Villamil, 2007). Estas posturas, incompatibles entre ellas, invitan a seguir indagando sobre la relación entre el vocabulario y las habilidades fonológicas. Dado que algunos de estos estudios han sido correlacionales, sería interesante ver si la promoción de habilidades fonológicas influye en el desarrollo del vocabulario de niños que están aprendiendo a leer.

Se tienen informes de la implementación de programas tendientes a promover habilidades inmersas en la conciencia fonológica; sin embargo, hay aspectos no explorados ampliamente. Su impacto en el vocabulario de los niños no se ha valorado justamente. Queda por aclararse el papel de la representación gráfica en el entrenamiento fonológico; algunos estudios sugieren que con su incorporación se obtienen mejores resultados en la lectura (Defior, 2008). Por otra parte, hay que señalar que estos entrenamientos, por lo general, son realizados en horas extraclase con grupos conformados por un reducido número de niños, $\mathrm{y}$ no se ha verificado su impacto cuando se desarrollan con grupos numerosos en ambientes áulicos cotidianos. La implementación de dichas actividades con niños que pertenecen a un estrato socioeconómico bajo, que por lo regular presentan un desarrollo deficiente en la lectura y escritura, se ha explorado poco (Diuk, Borzone y Ledesma, 2010). De ahí que el interés de este trabajo fuese implementar un programa con actividades favorecedoras de la conciencia fonológica como parte de las actividades cotidianas en un aula de primer grado escolar en una escuela donde asisten niños de un sustrato socioeconómico bajo.

Atendiendo este último punto y los anteriormente expuestos, la presente investigación tuvo como propósito evaluar los efectos de un programa diseñado para favorecer destrezas de conciencia fonológica sobre las habilidades psicolingüísticas, el vocabulario y las habilidades lectoras de niños que cursaban el primer grado de primaria. 
Para identificar el impacto del mencionado programa en los participantes, se observó el nivel inicial de sus habilidades fonológicas, sus destrezas psicolingüísticas y su vocabulario; después de la implementación del programa se identificaron de nuevo estos niveles y el de las habilidades lectoras adquiridas. También se identificaron las relaciones entre las distintas evaluaciones realizadas.

\section{MÉTODO}

\section{Participantes}

Participaron trece niños que conformaban un grupo natural de primer grado de educación primaria del turno matutino de una escuela del Estado de México. Seis eran hombres y el resto mujeres con edades entre seis y siete años. Ninguno era repetidor.

\section{Instrumentos}

Batería de Conciencia Fonológica. Se realizó una selección de la Batería de Conciencia Fonológica de Yáñez (2000) de la cual se eligieron tres procesos: 1) Discriminación Fonológica, 2) Análisis de palabras, compuesto por tres tareas: a) segmentación de palabras en sílabas, b) eliminación de un fonema y c) eliminación de una sílaba, y 3$)$ Análisis fonémico, que incluyó tres tareas: una destinada a identificar la sílaba inicial de las palabras y otra a identificar la rima. La mitad de los estímulos eran dibujos de palabras, y los restantes eran palabras presentadas oralmente por el examinador. Una tercera tarea consistía en presentar aisladamente los fonemas que conformaban una palabra, y el niño tenía que realizar la síntesis de los fonemas y construir la palabra correspondiente.

Test Illinois de Aptitudes Psicolingüiśticas. Adaptación Española del ITPA de Kirk, McCarthy y Kirk (Ballesteros \& Cordero, 2001). Está conformado por diez pruebas: comprensión auditiva, comprensión visual, asociación auditiva, asociación visual, expresión verbal, expresión motora, integración gramatical, integración visual, interacción auditiva y reunión de sonidos. Test de Vocabulario en Imágenes Peabody. (TVIP, Adaptación Hispanoamericana del Peabody Pictures Vocabulary Test, Dunn, Padilla, Lugo $\&$ Dunn, 1986). Evalúa el vocabulario receptivo o auditivo del individuo cuando este identifica los dibujos de las palabras que el examinador pronuncia.

Batería de evaluación para los procesos lectores de los niños de Educación Primaria (PROLEC). (Cuetos, Rodríguez \& Ruano, 2004). Se empleó la versión reducida recomendada por los autores, la cual explora cuatro procesos que intervienen en la comprensión del material escrito: Identificación de letras (igual-diferente), Procesos léxicos (lectura de pseudo palabras), Procesos sintácticos (estructuras gramaticales) y Procesos semánticos (comprensión de textos).

Programa de intervención en conciencia fonológica. Estuvo conformado por cuatro módulos: los tres primeros estuvieron constituidos por cinco sesiones, y el cuarto, por seis sesiones. En el primer módulo se fortaleció la segmentación silábica; se emplearon dibujos que representaban palabras de dos y tres sílabas. Se realizaba una actividad lúdica con dichos dibujos en la que el niño tenía que pronunciar la palabra y dividirla en sílabas. El objetivo del segundo módulo consistía en que los niños discriminaran y usaran la sílaba inicial de las palabras; para ello se realizaron dos sesiones de actividades con dibujos de palabras en las que se debía identificar la sílaba inicial, y dos sesiones en las que se debía decir la palabra sin pronunciar la primera sílaba; es decir, se omitía la primera sílaba de las palabras. Una última sesión se destinó a trabajar con las dos tareas, identificación y omisión de la sílaba inicial de las palabras. El tercer módulo se desarrolló de manera similar al segundo, solo que se trabajó con la sílaba final de las palabras. El cuarto módulo fue destinado a la síntesis fonémica; se realizaron cuatro sesiones en las cuales, a través de diversos juegos, se presentaban de manera aislada los fonemas que conformaban una palabra, y el niño debía construir la palabra correspondiente. Se incluyeron dos sesiones adicionales para repetir todas las actividades realizadas en los cuatro módulos con la intención de mejorar la ejecución de los niños en las diversas tareas fonológicas.

\section{Procedimiento}

Los instrumentos se aplicaron de manera individual en un lugar libre de distractores. En las dos primeras semanas se realizó la evaluación inicial en la que se aplicaron la Batería de Conciencia Fonológica, el ITPA y el Peabody. Posteriormente, se llevó a cabo la intervención, consistente en la aplicación del programa de manera colectiva en el salón de clases durante veintiuna sesiones semanales, distribuidas en seis meses. Al finalizar el programa, se llevó a cabo la segunda evaluación en la que se emplearon los mismos instrumentos que en la primera evaluación y además se aplicó el Prolec. Este instrumento requiere que los niños lean en tres de las cuatro pruebas que lo conforman. Por tal característica, no era posible que se aplicara antes de la intervención, la cual dio inicio al principio del primer ciclo escolar donde se recibe a los niños sin que hayan desarrollado la lectura. 


\section{RESULTADOS}

Los puntajes obtenidos en los instrumentos se presentan en dos secciones. En la primera se muestran los porcentajes de respuestas correctas en la pre y post evaluación para cada unos de los instrumentos. En la segunda sección se presenta la ejecución en cada uno de los instrumentos, diferenciándolos en dos grupos: los niños que terminaron leyendo al final del ciclo escolar y los niños que no lo lograron.

Análisis de las ejecuciones de la totalidad de la muestra en el pre y post test

Batería Conciencia Fonológica. En la primera evaluación, la discriminación fonológica y la identificación de rima de palabras representadas con dibujos, tarea perteneciente al análisis fonémico, tuvieron puntajes correctos superiores al $60 \%$ de los reactivos. Las tareas que presentaron más dificultad fueron la eliminación de fonemas y sílabas, que presentaron en promedio un $18 \%$ de respuestas correctas.
La más baja ejecución de todas las tareas fue la síntesis de fonemas con solo el $6.6 \%$ (véase Tabla1).

Después de la intervención, todas las ejecuciones en el postest, a excepción de la síntesis de fonemas, obtuvieron un incremento estadísticamente significativo. Aún así, las tareas de eliminación de fonemas y sílabas no alcanzaron el $60 \%$ de respuestas correctas.

Test de Vocabulario en Imágenes Peabody. De acuerdo con los parámetros del propio instrumento, las diversas puntuaciones obtenidas en la primera evaluación permiten ubicar a los niños con un nivel de vocabulario ligeramente por encima de lo esperado para su edad. Al finalizar la intervención, las puntuaciones disminuyen (véase parte superior Tabla 2), pero no de manera significativa.

Test Illinois de Aptitudes Psicolingüisticas (ITPA). Las puntuaciones totales de la prueba indican que los procesos implicados al captar, interpretar o transmitir un mensaje se encuentran en un nivel de normalidad. La aplicación del programa influyó solo en las puntuaciones directas pues incrementaron de manera significativa (parte inferior de la Tabla 2).

Tabla 1.

Número de reactivos y el porcentaje promedio de respuestas correctas en cada una de las pruebas de la Batería de Conciencia Fonológica, así como el nivel de significancia obtenido al comparar las medias de las puntuaciones del pretest y postest

\begin{tabular}{|c|c|c|c|c|c|c|c|}
\hline $\begin{array}{l}\text { CONCIENCIA } \\
\text { FONOLÓGICA }\end{array}$ & & & & Reactivos & Pretest & Postest & Significancia \\
\hline $\begin{array}{l}\text { Discriminación } \\
\text { Fonológica }\end{array}$ & & & & 42 & 87.5 & 97.6 & .004 \\
\hline \multirow{3}{*}{ Análisis de palabras } & Segmentación & & & 24 & 54.9 & 80.9 & .006 \\
\hline & Eliminación de fonemas & & & 20 & 14.1 & 36.2 & .019 \\
\hline & Eliminación de sílabas & & & 20 & 21.25 & 44.6 & .006 \\
\hline \multirow{5}{*}{ Análisis Fonémico } & Síntesis de fonemas & & & 22 & 6.6 & 10.4 & .688 \\
\hline & \multirow{4}{*}{ Categorización fonémica } & \multirow{2}{*}{$\begin{array}{l}\text { Identificación de sílaba } \\
\text { inicial de palabra }\end{array}$} & Dibujo & 9 & 51.9 & 88.8 & .008 \\
\hline & & & Palabra & 9 & 47.2 & 71.3 & .000 \\
\hline & & \multirow{2}{*}{ Identificación de rima } & Dibujo & 9 & 61.1 & 90.8 & .009 \\
\hline & & & Palabra & 9 & 42.3 & 69.4 & .008 \\
\hline
\end{tabular}

Tabla 2.

Promedio de las puntuaciones obtenidas en el vocabulario (Peabody) y en las habilidades psicolingüísticas (ITPA) en el pretest y postest, así como el nivel de significancia

\begin{tabular}{clccc}
\hline & & \multicolumn{2}{c}{ Evaluaciones } \\
\hline Instrumentos & \multicolumn{1}{c}{ Componentes } & Pretest & Postest & significancia \\
\hline \multirow{3}{*}{ Peabody } & Puntuación Directa & 62 & 62 & .897 \\
& Puntuación Estándar & 116.8 & 109.6 & .091 \\
& Rango Percentil & 79.2 & 66.3 & .061 \\
& Edad Equivalente & 8.3 & 7.9 & .395 \\
\hline \multirow{2}{*}{ ITPA } & Puntuaciones directas & 202.46 & 234.54 & .038 \\
& Puntuaciones típicas & 330.31 & 347.31 & .247 \\
\hline
\end{tabular}


Análisis de las ejecuciones de los niños lectores y no lectores

El programa de intervención terminó de implementarse dos meses antes de que concluyera el ciclo escolar; en ese momento, seis de los trece niños aún no leían. Por esa razón se analizaron de manera diferenciada las ejecuciones de los niños lectores y los no lectores.

Vocabulario. El análisis de las diversas puntuaciones del Peabody muestra que los niños que aún no leían, desde la primera evaluación presentaban puntajes menores que los que leían; no obstante, esta diferencia-analizada por medio de la prueba de Wilcoxon-no fue significativa. En el postest siguieron puntuando menos sin que la diferencia fuera significativa. Tampoco se encontraron diferencias significativas entre pre y post dentro de cada grupo.

Habilidades Psicolingüísticas. Los niños que leían, en un inicio tenían puntuaciones mayores en la suma total de las habilidades psicolingüísticas que los que no leían. En el postest la relación cambia, los niños no lectores superan a los lectores únicamente en las puntuaciones directas; en los dos momentos las diferencias, analizadas con la $\mathrm{U}$ de Mann-Whitney no fueron significativas. Un examen al interior de cada grupo entre la primera y segunda evaluación revela que solo en el grupo no lector hubo un incremento significativo en el total de las puntuaciones directas. Al revisar cada una de las diez habilidades psicolingüísticas, en el pretest los niños lectores tenían mejores puntuaciones en todas esas habilidades; para el postest, ese predominio solo persistía en la mitad de las habilidades, pues en el resto, los niños no lectores tuvieron puntuaciones mayores; no obstante, únicamente en la comprensión auditiva hay puntajes superiores en el grupo de niños no lectores $(p=$
.050). Un análisis al interior de cada grupo entre la primera y la segunda evaluación en cada una de las habilidades, revela que únicamente el grupo de niños no lectores mejoró sus puntajes en tres habilidades: memoria secuencial visomotora $(p=.027)$, integración gramatical $(p=.026)$ y expresión motora $(p=.045)$. Al agrupar las pruebas que valoraban el canal auditivo y el canal visual, se observó que en la segunda evaluación los niños no lectores superan a los lectores en el canal visual, aunque no significativamente.

Conciencia Fonológica. Los puntajes muestran diferencias entre los dos grupos de niños. En la Tabla 3 (columna 1) se observa cómo desde un inicio los lectores superaban a los no lectores en las pruebas de discriminación fonológica, eliminación de fonemas y eliminación de sílabas; dicha diferencia fue significativa de acuerdo con la $U$ de Mann-Whitney de muestras independientes. En el postest las diferencias continúan para la prueba de discriminación fonológica, en tanto que en las tareas de eliminación de fonemas y sílabas, en los niños que no leían, aunque tuvieron un número de respuestas menor en relación con los que sí leían, esta diferencia no fue significativa (véase Tabla 3, columna 2); pero en las actividades de identificación de sílaba inicial, las respuestas correctas de los niños no lectores se quedaron significativamente por debajo de quienes sí leían. La manera en que el programa influyó en cada grupo fue analizada con la prueba de Wilcoxon, que comparó las puntuaciones obtenidas en la evaluación pre y post al interior de cada grupo (véase Tabla 3, columna 3). Fueron cinco las tareas en las que se encontraron diferencias significativas en el grupo de no lectores, en tanto que para el grupo de lectores solo hubo diferencias en tres actividades.

Tabla 3.

Promedio de aciertos para cada una de las pruebas de conciencia fonológica obtenidas en el grupo de lectores y de no lectores durante el pretest y el postest

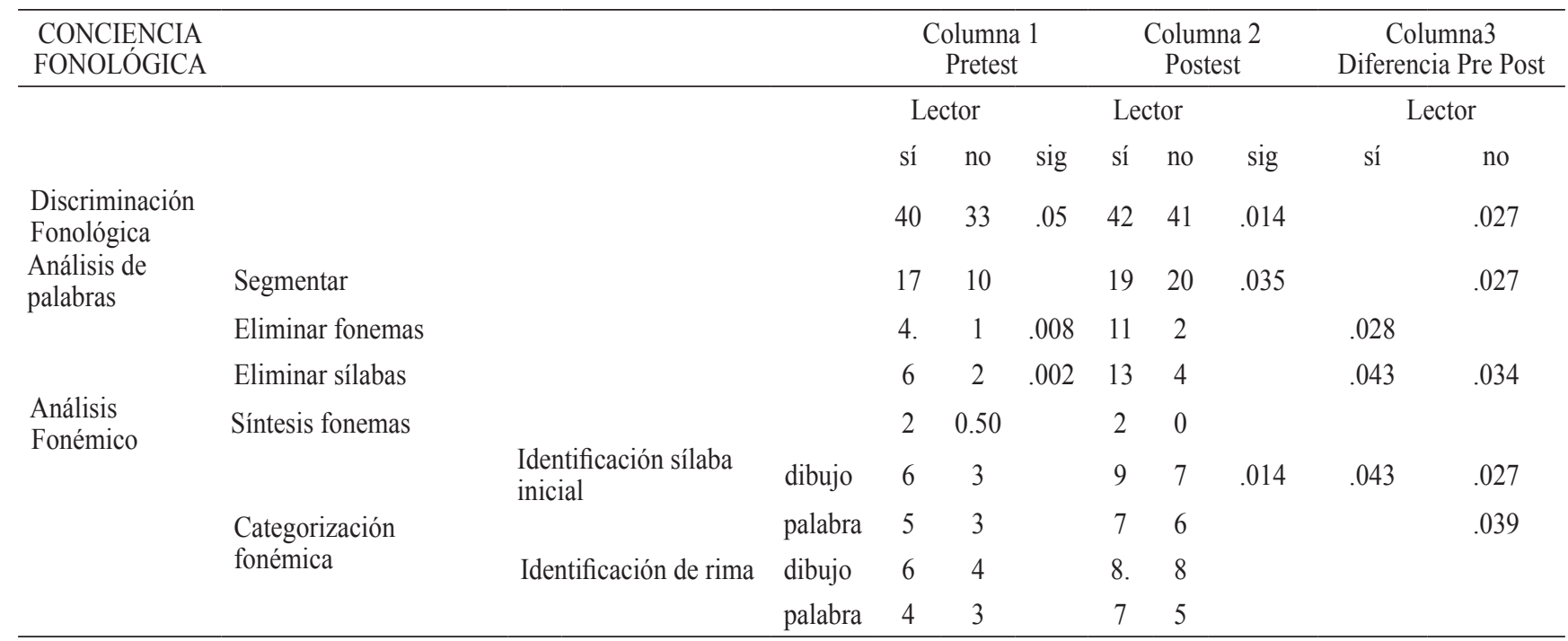

Nota. Se presentan únicamente los valores de probabilidad que resultaron menores al nivel de significancia de .05 al comparar el grupo de lectores y no lectores en la pre evaluación (columna 1), en la post evaluación (columna 2) y al comparar las puntuaciones de la pre y post evaluación en cada grupo (columna 3 ). 
Procesos Lectores. Solo la prueba de Igual-Diferente se aplicó a todos los niños. Resolver las pruebas restantes implicaba que los niños leyeran, por lo que las pruebas de Lectura de pseudo palabras, Estructuras gramaticales y Comprensión de textos solo se aplicaron a los niños que leían. A continuación se presentan los resultados de cada una de las pruebas:

Igual- Diferente: el promedio de respuestas correctas del grupo lector es de 17.12 y del no lector de 15.78 , sin diferencias significativas a través de la $\mathrm{U}$ de Mann Whitney $(p=.224)$; hay que hacer notar que cuatro niños que no sabían leer pudieron identificar visualmente en un nivel adecuado las semejanzas y diferencias en los pares de palabras, e incluso uno de ellos tuvo el más alto puntaje que se puede obtener en esta prueba.

Lectura de pseudo palabras: los puntajes totales menores a 16 indican dificultades en esta prueba; el promedio del grupo lector fue de 23.28, indicando con ello la inexistencia de problemas y que su ruta de lectura es fonológica.

Estructuras gramaticales: en general, las oraciones de mayor comprensión son las que tienen una estructura sintáctica activa; las dos estructuras restantes muestran un nivel menor de comprensión, pero similar entre ellas. Los puntajes totales menores a 6 indican dificultades y el promedio fue de 9.5.

Comprensión de textos: el promedio fue de 9, indicando con ello la inexistencia de dificultades; se observa que los textos expositivos obtuvieron los puntajes más bajos. En lo referente a las preguntas, las literales son más fáciles de responder sin que haya una diferencia importante entre ellas y las inferenciales.

Se realizaron análisis con la rho de Spearman para identificar las correlaciones entre los puntajes de los diversos instrumentos que se aplicaron. Con respecto al análisis realizado entre vocabulario y conciencia fonológica en la evaluación inicial no se encontraron correlaciones importantes y/o significativas. Fue en el postest donde se encontró una asociación significativa entre el vocabulario y la conciencia fonológica; para el caso de los niños lectores, el vocabulario se correlaciona con la identificación de la rima $(r=0.791, p=.032)$, y en los niños no lectores, con la identificación de sílaba inicial $(r=.971, p=.005)$. Para el caso del análisis del vocabulario y las habilidades psicolingüísticas, se detectaron asociaciones diferentes en el pretest y en postest. En la primera evaluación, los niños lectores presentaron una asociación entre los puntajes de vocabulario con la asociación visual $(r=.753, p=.031)$ y la integración gramatical $(r=.783, p=.032)$, y los niños no lectores presentaron una asociación estrecha entre la asociación auditiva y el vocabulario $(r=.975, p=.005)$. En los datos generales del postest se notó que el vocabulario correlacionó con la suma de los puntajes de las habilidades del canal auditivo $(r=.667, p=.013)$ y un análisis más detallado indica que es la comprensión auditiva con la que se asocia de manera moderada y significativa $(r=.625, p=$ .017). No se encontró ninguna correlación significativa al interior de cada grupo.

En la Tabla 4 se presentan las asociaciones que resultaron significativas entre conciencia fonológica y habilidades psicolingüísticas. En general, en los niños lectores se encuentran más elementos que correlacionan, siendo la memoria secuencial auditiva la que correlaciona con más habilidades fonológicas en el pretest. En el postest, los elementos que correlacionan son diferentes. En lo que concierne a los niños no lectores solo se encuentra correlación entre la comprensión auditiva y la segmentación en el pretest.

Tabla 4.

Correlaciones entre las habilidades fonológicas y psicolingüísticas en el pretest y postest para niños lectores y no lectores

\begin{tabular}{lcccr}
\hline \multicolumn{1}{c}{ CONCIENCIA FONOLÓGICA } & \multicolumn{2}{c}{ Habilidades Psicolingüísticas } \\
\hline & \multicolumn{2}{c}{ Lector } & No Lector \\
\cline { 2 - 5 } & Pretest & Postest & Pretest \\
\cline { 2 - 5 } & Memoria auditiva & $\begin{array}{c}\text { Expresión } \\
\text { verbal }\end{array}$ & $\begin{array}{c}\text { Integración } \\
\text { gramatical }\end{array}$ & $\begin{array}{c}\text { Comprensión } \\
\text { auditiva }\end{array}$ \\
\cline { 2 - 5 } Discriminación & $\mathrm{r} / \mathrm{p}$ & $\mathrm{r} / \mathrm{p} / \mathrm{p}$ & $\mathrm{r} / \mathrm{p}$ \\
Segmentación & $.760 / .048$ & $.798 / .031$ & $.845 / .034$ \\
Síntesis fonemas &. & & \\
Identificación sílaba palabra & $.765 / .045$ & & $.869 / .028$ \\
Eliminar fonema & $.767 / .044$ & & \\
\hline
\end{tabular}

Nota. $r$ : rho de Spearman, $p$ : nivel de significancia 
Posteriormente, se identificaron correlaciones únicamente para los niños del grupo lector entre las pruebas del Prolec y las puntuaciones obtenidas en la batería de conciencia fonológica. En la Tabla 5 se presentan solo las que resultaron significativas, advirtiéndose que son moderadas, principalmente entre la comprensión de textos y la identificación de sílaba inicial y rimas cuando éstas son presentadas por medio de dibujos. Por otra parte, la comprensión de estructuras gramaticales se relacionó exclusivamente con la identificación de la sílaba inicial, aun cuando las palabras eran presentadas oralmente y con dibujos. Hay que destacar que la lectura de pseudopalabras no correlacionó con ninguna tarea de conciencia fonológica, pero sí correlacionó con otra tarea de lectura, la comprensión de textos $(\mathrm{r}=.550, \mathrm{p}=034)$. También se buscaron correlaciones entre las puntuaciones del Prolec y las habilidades psicolingüísticas, donde únicamente la habilidad de integración visual se asocia en un nivel alto con la identificación visual de palabras iguales y diferentes, así como con la comprensión de textos y de estructuras gramaticales. La correlación entre los diversos elementos del Prolec y el vocabulario receptivo solo se manifestó de manera moderada entre este último y la comprensión de textos.

\section{DISCUSIÓN}

En general, puede decirse que los niños ingresan con un nivel de habilidades psicolingüísticas y de vocabulario considerados dentro de la norma de acuerdo con los ins- trumentos empleados. Se considera que este nivel ha sido obtenido por la estimulación en el hogar y en la educación preescolar, y que de acuerdo con este, los niños tienen las habilidades comunicativas indispensables para captar, interpretar y/o transmitir un mensaje, lo que sugiere que no tienen problemas comunicativos que los condujeran a presentar problemas de aprendizaje. Su nivel de vocabulario también cae dentro de la norma, y en determinado momento, de acuerdo con los planeamientos de Walley, Metsala y Garlock (2003), constituiría una influencia moderada para el proceso de lectura, en particular en la comprensión de la misma.

Esta ventaja en vocabulario y habilidades psicolingüísticas no se presenta en las habilidades fonológicas; desde un comienzo, los niños que culminaron adecuadamente el proceso de lectoescritura mostraban una ventaja significativa en las actividades de discriminación fonológica, eliminación de fonemas y eliminación de sílabas, en relación con los niños que no adquirieron la lectoescritura. El buen desarrollo de tales habilidades en los niños lectores desde un inicio, hace suponer que estos las han ejercitado de alguna manera que pudiera estar relacionada, por ejemplo, con las actividades realizadas en su hogar o en el jardín de niños de procedencia; se tiene evidencia de que algunas de las habilidades contempladas en la conciencia fonológica se pueden desarrollar en la edad preescolar, antes del aprendizaje de la lectoescritura (Herrera, Defior \& Lorenzo, 2007; Porta, 2008).

Tabla 5.

Correlaciones entre las puntuaciones de los procesos lectores, el vocabulario y las habilidades fonológicas y psicolingüísticas de los niños lectores

\begin{tabular}{|c|c|c|c|c|c|c|c|}
\hline \multirow[b]{3}{*}{ Conciencia Fonológica } & & \multicolumn{6}{|c|}{ Procesos Lectores } \\
\hline & & \multicolumn{2}{|c|}{ Estructuras gramaticales } & \multicolumn{2}{|c|}{ Igual/diferente } & \multicolumn{2}{|c|}{ Comprensión de textos } \\
\hline & & $\mathrm{r}$ & $\mathrm{p}$ & $\mathrm{r}$ & $\mathrm{p}$ & $\mathrm{r}$ & $\mathrm{p}$ \\
\hline \multirow{2}{*}{ Identificación de sílaba inicial } & palabras & .670 & .048 & & & & \\
\hline & dibujos & .689 & .040 & & & .679 & .044 \\
\hline Identificación de rimas & dibujos & & & .786 & .012 & .779 & .013 \\
\hline \multicolumn{8}{|l|}{ Habilidades Psicolingüísticas } \\
\hline Integración visual & & .731 & .040 & .723 & .043 & .854 & .007 \\
\hline Vocabulario & & & & & & .663 & .050 \\
\hline
\end{tabular}

Nota. $r$ : rho de Spearman, $p$ : nivel de significancia 
De acuerdo con la literatura, las habilidades comprendidas en la conciencia fonológica se ven fortalecidas, tanto por ejercicios exprofeso (Lorenzo, 2001), como por el proceso mismo de la adquisición de la lectoescritura (Porta, 2008). El programa de entrenamiento en conciencia fonológica implementado en este estudio ayudó a los niños que ya tenían cierto desarrollo en esas habilidades, principalmente en el análisis de palabras, pues las diferencias significativas entre la evaluación pre y post de los niños lectores, se encontraron en la eliminación de fonemas y la eliminación de sílabas. También les ayudó en el análisis fonémico en lo que corresponde a la identificación de la sílaba inicial de las palabras cuando estas son presentadas oralmente. El alto nivel obtenido en esas habilidades en un inicio y las fortalecidas con el programa es lo que posiblemente los llevó a culminar adecuadamente el proceso de lectoescritura. El hecho de que los niños con mejores puntajes en la prueba de conciencia fonológica terminaran el curso leyendo, constituye una evidencia para las propuestas de que la conciencia fonológica es una variable predictiva del rendimiento lector (Lorenzo, 2001; Bravo, Villalón y Orellana, 2006).

El programa con el cual se trabajó parece favorecer en especial a los niños no lectores, pues se vieron mejoras significativas en la post evaluación en más tareas que en el grupo lector. Esas mejorías se presentaron en la discriminación fonológica, en el proceso de análisis de palabras en las tareas de segmentación y eliminación de sílabas, y en el análisis fonémico en la identificación de la sílaba inicial de las palabras, ya fueran presentadas oralmente $o$ por medio de dibujos.

Si bien se puede decir que el programa ayudó de manera general a fortalecer las habilidades comprendidas en la conciencia fonológica de todos los niños, hay que reconocer que lo hace diferencialmente de acuerdo con sus habilidades fonológicas previas y sus competencias lectoras. Los niños que no han desarrollado dichas habilidades y competencias se vieron favorecidos por el programa en más tareas relacionadas con la conciencia fonológica, pero podría suponerse que no lo suficiente, pues no lograron altos puntajes en la evaluación de conciencia fonológica ni adquirieron las competencias lectoras. En el programa se incorporaron las principales tareas incluidas en la conciencia fonológica; no obstante, un análisis a posteriori revela que se realizaron más sesiones que ejercitaban las unidades silábicas y menos sesiones para ejercitar unidades fonémicas. También es importante señalar que los elementos visuales que acompañaron los elementos fonológicos siempre fueron dibujos, no como en el caso del trabajo de Defior (2008) en el que los elementos visuales eran letras o sílabas. En este caso no se realizó así para no interferir con el método de enseñanza de la lectoescritura empleado por la profesora del grupo.

Es probable que si hubiesen existido más sesiones en general, pero en especial las de unidades fonémicas y con asociaciones con las grafías, los niños que iniciaron con habilidades fonológicas poco desarrolladas hubiesen podido verse favorecidos más ampliamente en ellas y avanzar también en el proceso de adquisición de la lectura. Se tiene evidencia de que las dificultades para realizar tareas con los fonemas predicen problemas en la lectura y la escritura (Bravo, Bermeosolo \& Pinto, 1992; Porta 2008) y que la asociación de fonemas con grafías en la estimulación fonológica ayuda a la lectoescritura (Defior, 2008). De ahí que se proponga realizar otros estudios, con más actividades y sesiones que se enfoquen en las unidades fonémicas y dónde se evalúe el papel de las grafías.

Los datos parecen indicar que el nivel de ejecución de discriminación fonológica que desde un inicio presentaban los niños que terminaron el curso con la competencia lectora en un nivel adecuado pudiera ser una variable involucrada en el buen desarrollo de la misma. El que los niños desde un principio distinguieran auditivamente de manera correcta si un par de palabras (baño-paño, por ejemplo) eran iguales o diferentes, estaría indicando una habilidad para distinguir unidades mínimas pronunciables y constituyentes de una palabra, habilidad que favoreció para que tanto en el pretest como en el postest ellos obtuvieran mejores puntajes en la eliminación de fonemas y eliminación de sílabas. Aunque el programa de intervención empleado en este trabajo ayudó significativamente a los niños no lectores en tales actividades, estos nunca alcanzaron en el postest niveles parecidos a los niños lectores. Evidentemente, pueden estar involucrados otros factores, pero al menos para la población evaluada pareciera que se hubiese requerido estimular de alguna manera las habilidades para reconocer, identificar y manipular las unidades fonológicas de la palabra desde la edad preescolar, para que cuando se enfrentaran a las tareas de adquisición de la lectura proporcionadas en la institución escolar, estas, en conjunto con un cierto nivel de habilidades fonológicas, los hubiese llevado a poseer un adecuado nivel de competencia lectora.

En los niños lectores se observa una asociación positiva entre las puntuaciones obtenidas en algunas tareas de conciencia fonológica del postest y las puntuaciones de la prueba de lectura. De manera general, se puede decir que la identificación de sílabas iniciales de las palabras y la identificación de rimas se asociaron con una buena comprensión de enunciados con diversas estructuras gramaticales y de diversos tipos de textos. Estos datos 
apoyan la propuesta de que en la comprensión lectora se encuentran involucrados los procesos de decodificación fonológica y fonémica (Bravo, Bermeosolo \& Pinto, 1992). Un dato que se reporta en la literatura es que los buenos lectores no tienen dificultad en la lectura de pseudo palabras (Lorenzo, 2001) e incluso algunos investigadores toman la ejecución de esta última como un predictor de la comprensión de textos (Porta, 2008). En este caso se obtuvo evidencia semejante: los puntajes de estos dos elementos, la lectura de pseudopalabras y la comprensión de textos obtenidos por el mismo instrumento (Prolec) presentaron una correlación significativa.

Los niños que no aprendieron a leer presentaron ejecuciones altas en la tarea visual de identificar si las palabras eran iguales o diferentes, lo cual sugiere que en ellos es en el proceso de discriminación auditiva donde puede existir un déficit, ya que no tienen buenos puntajes en las actividades fonológicas, en especial cuando se trabaja con fonemas. Lo anterior se corrobora al realizar un análisis de las puntuaciones de sus habilidades psicolingüísticas atendiendo a los canales perceptivos, el cual muestra que en estos niños las puntuaciones obtenidas en el procesamiento de información, que involucra preferencialmente al canal auditivo, son ligeramente menores que en el canal visual. La tendencia a encontrar un déficit en el aspecto auditivo por medio de diferentes instrumentos fortalece la idea de que, en este caso, el que los niños no adquirieran la lectoescritura involucró, entre otros, un factor relativo a la falta de ejercitación de la discriminación auditiva. No se puede descartar que los niños que no progresaron en la lectura persistieran en mantener con cierta rigidez las estrategias para el procesamiento del texto, dando prioridad a los aspectos visuales en los que estaban más desarrollados y dejando de lado los auditivos. Esta posibilidad existe porque de acuerdo con Bravo, Bermeosolo y Pinto (1992) los niños que tienen bajo rendimiento verbal, problemas lectores y/o presentan dislexia, presentan cierta inflexibilidad para emplear estrategias alternativas en la superación de sus dificultades.

Por otra parte, el que los niños estén expuestos a un programa que estimula habilidades fonológicas parece influir en su vocabulario, debido a la asociación hallada entre dichos elementos. En la evaluación inicial no se encontraron correlaciones entre las habilidades fonológicas y el vocabulario en ninguno de los dos grupos. No obstante, los datos obtenidos al término del programa revelan que existe una correlación alta y significativa entre el vocabulario receptivo y las tareas de análisis fonémico de la conciencia fonológica, lo que coincidiría con los hallazgos de Diuk, Borzone y Ledesma (2010) y Maciá (2012). Hay que precisar que esto ocurre de manera diferencial en función de si se tiene o no la competencia lectora. Si los niños ya leen, la asociación se establece entre el vocabulario y la identificación de rimas, en tanto que si no leen, la relación es entre el vocabulario y la identificación de la sílaba inicial. Habrá que indagar más ampliamente si siempre se dan estos efectos diferenciales. Un punto a resaltar es el hecho de que la asociación entre vocabulario y habilidades fonológicas se estableciera solo después del entrenamiento, lo que invita a considerar el tipo de relación entre estos elementos. Márquez y De la Osa (2003) argumentan que la relación establecida era unidireccional, siendo el vocabulario el que interviene en la conciencia fonológica. Los hallazgos de este estudio parecieran indicar que la conciencia fonológica también influye en el vocabulario o bien pudiera interpretarse de acuerdo con lo expuesto por Vargas y Villamil (2007), que la relación entre esos dos elementos se establece cuando se domina la alfabetización. No obstante, esto último aplicaría a las correlaciones encontradas en los niños lectores, pero no explicaría por qué en los niños no lectores se establecieron las correlaciones. Lo anterior habrá de esclarecerse en otros estudios donde se controle el nivel de alfabetización y la exposición a tareas en donde se vea implicada la conciencia fonológica. Así mismo, se sugiere emplear otros instrumentos que valoren el vocabulario tanto como las habilidades fonológicas.

Considerando que el desarrollo de las habilidades psicolingüísticas y fonológicas se inicia en momentos anteriores a la instrucción escolar y en contextos diferentes a la escuela, se analizaron las correlaciones entre ellas, y se observó que la memoria auditiva se asocia con diversas habilidades fonológicas: la discriminación fonológica, la identificación de la sílaba inicial de las palabras y la síntesis de fonemas, siendo ésta última la tarea más difícil, pero solo con los niños lectores. Lo anterior sugiere que los ejercicios de memoria auditiva podrían ayudar a mejorar las habilidades fonológicas y repercutir en la adquisición de la lectura. Parece haber poca influencia del entrenamiento fonológico en las habilidades psicolingüísticas, pues las puntuaciones de éstas últimas permanecieron prácticamente sin cambio al final de dicho entrenamiento; únicamente los niños no lectores mejoraron en la memoria visomotora, en la integración gramatical y en la expresión motora, y coincidentemente en estos niños no se encontraron correlaciones entre esos dos tipos de habilidades en el postest. Habrá que investigar más detalladamente cómo se manifiestan las relaciones entre las habilidades en cuestión.

Los datos derivados de este estudio, aunados con los presentados en la literatura, permiten consolidar la idea de que los déficits en la conciencia fonológica dificultan el establecimiento de las correspondencias grafema-fonema, necesarias para la adquisición de la lectoescritura. Hay que 
destacar que esta última no es un proceso unitario, pues en él se encuentran implicados procesos dependientes de habilidades fonológicas; de ahí que algunos postulen que la habilidad de discriminación fonológica es fundamental para el adecuado manejo del código alfabético y, con ello, para el establecimiento adecuado de la lectura. Por otra parte, se pone de manifiesto que una estimulación diversa del lenguaje oral brindada al niño de manera previa a la instrucción de la lectoescritura resulta importante para que esta llegue a un nivel adecuado y le posibilite al niño terminar el primer grado escolar con una competencia lectora acorde a ese grado.

\section{REFERENCIAS}

Arnaiz, P., Castejón, J. L. \& Ruiz, M.S. (2002). Influencia de un programa de desarrollo de habilidades psicolingüísticas en el acceso a la lectoescritura. Revista de Investigación Educativa, 20 (1), 189-208.

Ballesteros, S. \& Cordero, A. (2001) Test Illinois de Aptitudes Psicolingüisticas. Adaptación española del Illinois Test of Psycholinguistic Abilities de Kirk, S., McCarthy, J. y Kirk, W.: Madrid, TEA.

Bravo, L. (2004). La conciencia fonológica como una posible zona de desarrollo próximo para el aprendizaje de la lectura inicial. Revista Latinoamericana de Psicología, 36, 1, 21-32.

Bravo, L., Bermeosolo, J. \& Pinto, A. (1992). Procesamiento auditivo-fonémico y comprensión lectora silenciosa en un grupo de disléxicos. Revista Latinoamericana de Psicología, vol. 24, núm. 3 , pp. 275-292.

Bravo, L., Villalón, M. y Orellana, E. (2006). Diferencias en la predictividad de la lectura entre primer año y cuarto año básicos. Psykhe, 15 (1), 3-11

Cuadro, A. \& Trías, D. (2008). Desarrollo de la conciencia fonémica. Revista Argentina de Neuropsicología, 11, 1-8.

Cuetos, F., Rodríguez, B \& Ruano, E, (2004). Evaluación de los procesos lectores. PROLEC. Madrid, TEA.

Dalurzo, M. J. \& González, L. A. (2004) El vocabulario y el rendimiento académico en G. Vázquez (Coord.). Español con fines académicos: de la comprensión a la producción de textos. Madrid, Edinumem. 87-97

Defior, S. (2008). Facilitar el aprendizaje inicial de la lectoescritura. Papel de las habilidades fonológicas. Infancia y Aprendizaje, 31, 3, 333-354

Diuk, B; Borzone, A. M. y Ledesma, R. (2010). Conocimiento de vocabulario, representaciones fonológicas y sensibilidad en niños pequeños de distinto sector social de procedencia. SUMMA Psicológica, 7 (1), 33- 50.

Dunn, L., Padilla, I, Lugo, D. \& Dunn, L. (1986) Test de Vocabulario en Imágenes Peabody. (TVIP) Adaptación Hispanoamericana del Peabody Pictures Vocabulary Test. Minenesota: American Guidance Service, Publisher's Building.
Flórez-Romero, R, Restrepo, M. A. \& Schwanenflugel, P. (2009). Promoción del alfabetismo inicial y prevención de las dificultades en la lectura: Una experiencia pedagógica en el aula de preescolar. Avances en Psicología Latinoamericana, 27 (1), 79-96.

González, M. J. \& Delgado, M. (2009). Rendimiento académico y enseñanza-aprendizaje de la lectoescritura en educación infantil y primaria: un estudio longitudinal. Infancia y Aprendizaje, 32, 3, 265-276

Herrera, V. (2005). Habilidad lingüística y fracaso lector en los estudiantes sordos. Estudios Pedagógicos, 31, 2, 121-135.

Herrera, L., Defior, S. \& Lorenzo, O. (2007). Intervención educativa en conciencia fonológica en niños prelectores de lengua materna española y tamazight. Comparación de dos programas de entrenamiento. Infancia y Aprendizaje, 2007, 30 (1), 39-54.

Hesketh, A., Adams, C. \& Nightingale, C, (2000). Metaphonological Abilities of Phonologicallly Disordered Children. Educational Psychology, 20, 24, 483-498.

Jiménez, J.E. \& Ortiz, M. R. (2000). Conciencia metalingüística $\mathrm{y}$ adquisición lectora en la lengua española. The Spanish Journal of Psychology, 3 81), 37-46.

Kavale, K. (1981). Functions of the Illinois Test of Psycholinguistic Abilities (ITPA): Are they trainable? Exceptional Children, 47 (7), 496-510.

Kirk, S. A., McCarthy, J. J. \& Kirk, W. D.(1961). The Illinois Test of Psycholinguistic Abilities, Urbana 111, 1961 (rev. 1968), Univ. of Illinois Press.

Lorenzo, J. R. (2001) Procesos cognitivos básicos relacionados con la lectura. Primera parte: La conciencia fonológica. Interdisciplinaria, 18 (1) 1-33.

Macià, M (2012). Vocabulario y Conciencia Fonológica. Tesis de Maestría en Educación Infantil. Universidad Internacional de la Rioja. Facultad de Educación. Barcelona.

Márquez, J. \& De La Osa, P. (2003). Evaluación de la conciencia fonológica en el inicio lector. Anuario de Psicología, 34 (3), 357-370.

Moraleda, M. (1991). Relación entre el desarrollo de las funciones verbales y el rendimiento académico en los alumnos de educación primaria. Revista Complutense de Educación, 2, (1), 93-110.

Ninio, A. \& Bruner, J. (1978). The Achievement and Antecedents of Labeling. Journal of Child Language, 5, 1-15.

Palomino, J. (2011) Comprensión lectora y rendimiento escolar, una ruta para mejorar la comunicación.COMUNIC@CCION: Revista de Investigación y Desarrollo, 2, 2, 27-

Passenger, T., Stuart, M. \& Terrel, C. (2003). Phonological Processing and Early Literacy. Journal of Research in Reading, 23 (1), 55-66.

Poe, M. D., Burchinal, M. R. \& Roberts,J.E, (2004). Early Language and the Development of Children's Reading Skills. Journal of School Psyhology, 42, 4315-332 
Porta, M. (2008). Comprendiendo el rol predictivo de la conciencia fonológica en la adquisición de la lectura y en la detección de niños en riesgo pre-lector. Revista de Orientación Educativa 4 87-103

Santiuste, V. \& López, C. (2005). Nuevos aportes a la intervención en las dificultades de lectura. Universitas Psichological, 4, 1,

Savage, R. S. \& Carles, S. (2004). Predicting Curriculum and Test Performance at Age 7 Years from Pupil Background Baseline Skills and Phonological Awareness at Age 5. British Journal of Educational Psychology, 74, 155-171.

Sénéchal, M., Thomas, E. \& Monker, J. (1995). Individual Differences in 4-Year-old Children's Acquisition of Vocabulary During Storybook Reading. Journal of Educational Psychology, 87, 2, 218-229.

Stahl, S. \& Yaden, D. (2004). The Development of Literacy in Preschool and Primary Grades: Work by the Center for the Improvement of Early Reading Achievement, The Elementary School Journal,. 105, (2), 141-165

Treiman, R. (1991). Phonological Awareness and its Roles in Learning to Read and Spell. En D. J. Sawyer y B.J. Fox (Eds.) Phonological Awareness in Reading. The Evolution of Current Perspective (pp.159-189) New York: Springer Verlag.
Valdez_Menchaca, M. \& Whitehurst, G.J. (1992). Accelerating Language Development Through Picture-book Reading: A Systematic Extension to Mexican Day Care. Development Psychology, 28, 6, 1106-1114.

Vargas, A. \& Villamil, W. (2007). Diferencias en el rendimiento lector entre dos grupos de niños de transición debidas a una intervención promotora del alfabetismo emergente en el aula. Revista Colombiana de Psicología, 16, 65-76.

Walley, A., Metsala, J. \& Garlock, V. (2003). Spoken vocabulary growth: Its role in the development of phoneme awareness and early reading ability. Reading and Writing, 16, 5- 20.

West, R. F., Stanovich, K. E. \& Mitchell, H. R. (1993). Reading in the Real World and its Correlates. Reading Research Quaterly, 28, 34-51.

Wood, C.L. \& Terrell, C. (2000). Rhyme awareness, orthographic analogy use, phonemic awareness and reading: an examination of relationships. Educational Psychology, 20 (1), 5-17.

Yáñez, G. (2000). Batería neuropsicológica para la evaluación de niños con trastornos del aprendizaje: Estandarización con niños de la zona metropolitana de la Ciudad de México. Disertación doctoral no publicada, Universidad Nacional Autónoma de México, México. 